\title{
Atomic T Tauri disk winds heated by ambipolar diffusion
}

\section{Observational tests}

\author{
P. J. V. Garcia ${ }^{1,2, \star}$, S. Cabrit ${ }^{3}$, J. Ferreira ${ }^{4}$, and L. Binette ${ }^{5}$ \\ 1 Centro de Astrofísica da Universidade do Porto, Rua das Estrelas, 4150-762 Porto, Portugal \\ 2 CRAL/Observatoire de Lyon, CNRS UMR 5574, 9 avenue Charles André, 69561 St. Genis-Laval Cedex, France \\ 3 Observatoire de Paris, DEMIRM, UMR 8540 du CNRS, 61 avenue de l'Observatoire, 75014 Paris, France \\ 4 Laboratoire d'Astrophysique de l'Observatoire de Grenoble, BP 53, 38041 Grenoble Cedex, France \\ ${ }^{5}$ Instituto de Astronomía, UNAM, Ap. 70-264, 04510 D. F., México
}

Received 18 July 2001 / Accepted 2 August 2001

\begin{abstract}
Thermal and ionization structures of self-similar, magnetically-driven disk winds obtained in a companion paper (Garcia et al. 2001) are used to compute a series of jet synthetic observations. These include spatially resolved forbidden line emission maps, long-slit spectra, as well as line ratios. Line profiles and jet widths appear to be good tracers of the wind dynamics and collimation, whereas line ratios essentially trace gas excitation conditions. All the above diagnostics are confronted to observations of T Tauri star microjets. Convolution by the observing beam is shown to be essential for a meaningful test of the models. We find that jet widths and qualitative variations in line profiles with both distance and line tracers are well reproduced. A low-velocity [O I] component is also obtained, originating from the disk wind base. However, this component is too weak, predicted maximum velocities are too high and electronic and total densities are too low. Denser and slower magnetized winds, launched from disks with warm chromospheres, might resolve these discrepancies.
\end{abstract}

Key words. ISM: jets and outflows - stars: pre-main sequence - MHD - line: profiles - accretion disks

\section{Introduction}

Jets from young protostars such as T Tauri stars remain an enigma. Indeed, despite tremendous efforts, both observationally and theoretically, we do not know whether jets are driven by the disk alone (so called "disk winds"), the central protostar alone ("stellar winds") or the interaction zone between the disk inner edge and the protostellar magnetosphere ("X-winds"). As argued in Garcia et al. (2001, hereafter Paper I) probing the ultimate energy source powering $\mathrm{T}$ Tauri jets requires reliable observational predictions from any dynamical jet model. To do so, the thermal and ionization states of the gas must then be solved along the flow. This remains to be addressed in a fully self-consistent way, leaving room for speculations.

In contrast with recent approaches which assumed a constant temperature and ionization profile (Shang et al. 1998; Cabrit et al. 1999), in Paper I we solved a posteriori

Send offprint requests to: P. J. V. Garcia,

e-mail: pgarcia@astro.up.pt

* CAUP Support Astronomer, during year 2000, at the Isaac Newton Group of Telescopes, Sta. Cruz de La Palma, Spain. the thermal and ionization structures of a magneticallydriven disk wind heated by ambipolar diffusion. Indeed, of all possible heating mechanisms only ambipolar diffusion heating allows "minimal" thermal solutions, in the sense that the same physical process - non-vanishing currents is responsible for both jet dynamics and heating. As a consequence no additional tunable parameter is invoked for the jet thermal description. We therefore extended the pioneering work of Safier (1993a,b) by (1) using magneticallydriven (cold) jet solutions, self-consistently computed with the underlying accretion disk Ferreira (1997); (2) a more accurate treatment of ionization using the Mappings Ic code and ion-neutral momentum exchange rates including the thermal contribution.

As in Safier (1993a), we obtained jets with a temperature plateau around $10^{4} \mathrm{~K}$, but ionization fractions revised downward by a factor of 10-100. This last fact is due to the previous omission of thermal speeds in ion-neutral momentum-exchange rates and to our different jet solutions. Moreover, the physical origin of the hot temperature plateau has been shown to represent a robust property of magnetically-driven disk winds heated by ambipolar 
diffusion. Last but not least, we showed that the calculated thermal and ionization states remain consistent with the approximations used for jet calculations (see Appendix C, Paper I): (1) single fluid description of a (2) thermalized (all chemical species share the same temperature), (3) cold (negligible thermal pressure gradient) and (4) perfectly conducting plasma. We stress that such consistency checks are of an extreme physical importance and must therefore be made.

In this paper, we compare the detailed model calculations obtained in Paper I with observations of forbidden line emission regions in $\mathrm{T}$ Tauri stars, believed to probe accretion-driven winds (Cohen et al. 1989; Cabrit et al. 1990; Hartigan et al. 1995, hereafter HEG95). The high-velocity emission has been spatially resolved in a dozen stars, and found to arise from collimated "microjets" extended over only a few 100 AU (Solf 1989; Kepner et al. 1993; Hirth et al. 1994a,b; Burrows et al. 1996; Ray et al. 1996; Hirth et al. 1997; Bacciotti \& Eislöffel 1999; Lavalley-Fouquet et al. 2000; Dougados et al. 2000a; Bacciotti et al. 2000, and Refs. therein). These microjets represent currently our best opportunity to test accretiondriven jet models: (1) At such small distances from the star, the intrinsic jet structure is (hopefully) less perturbed by instabilities or interactions with the ambient medium than in the more distant jet sections accessible in infrared sources; (2) Direct, albeit uncertain, estimates for the disk accretion rate have become recently available from measurements of continuum excess in the stellar photospheric spectrum (HEG95, Gullbring et al. 1998).

We point out that the "correct" jet model should pass all available observational tests, namely: microjet widths as measured by adaptive optics and the Hubble Space Telescope, long slit spectra, and integrated line profiles, line fluxes and line ratios. Note that only the latter constraints based on integrated profiles were available to Safier (1993b). We are now in a position to perform more detailed tests of the class of self-similar, magnetically driven cold disk winds, involving spatially-resolved diagnostics. We also stress that given the steep gradients in physical conditions at the base of the wind, meaningful comparison with observations requires proper convolution of maps and long-slit spectra by the observing beam.

This article is structured as follows. Section 2 is devoted to the predicted forbidden line emission diagnostics and their comparison with T Tauri stars observations. We conclude in Sect. 3 by summarizing the successes and failures of our model, and by discussing perspectives to resolve the discrepancies.

\section{Predicted forbidden line emission and comparison with $T$ Tauri microjets}

We used three different models of self-similar magnetized accretion-ejection structures (MAES, Ferreira 1997), labelled A, B and C. They are mainly characterized by a different ejection index

$\xi=\frac{\mathrm{d} \ln \dot{M}_{\mathrm{acc}}(\varpi)}{\mathrm{d} \ln \varpi}$

measuring the local ejection efficiency, where $\dot{M}_{\text {acc }}$ is the disk accretion rate at the cylindrical radius $\varpi$. Note that $\xi=0$ in a standard accretion disk. In our models, the ejection index controls the jet opening, a smaller $\xi$ providing a larger jet radius. Model $\mathrm{A}$ is obtained for $\xi=0.01$, model $\mathrm{B}$ for $\xi=0.007$ and model $\mathrm{C}$ for $\xi=0.005$. A magnetized accretion-ejection structure is assumed to be settled around a protostar of $M_{*}=0.5 M_{\odot}$, between an inner and an outer edge of, respectively, $\varpi_{\mathrm{i}}=0.07 \mathrm{AU}$ and $\varpi_{\mathrm{e}}=1 \mathrm{AU}$. Outside this radius, we assume the presence of a standard accretion disk fueling the MAES with mass (and possibly magnetic flux). The accretion rate is taken to vary between $10^{-8}$ and $10^{-5} M_{\odot} \mathrm{yr}^{-1}$. We refer the reader to Sect. 2 in Paper I for further details concerning the dynamical models and for a discussion of our choice of parameters.

\subsection{Calculation of synthetic emission maps and profiles}

Synthetic emission maps and long slit spectra along the jet axis were computed in the lines of [O I $] 6300$, [S II]6731, H $\alpha$, and [N II]6584, using the emissivities provided by Mappings Ic. Emission was assumed optically thin, simplifying calculations of the emergent profiles. This assumption is clearly adequate for forbidden lines, though possibly not for $\mathrm{H} \alpha$ whose opacity is probably dominated by scattering of Ly $\alpha$ photons. Our results for the latter line are therefore only indicative, a complete treatment of the line radiative transfer being outside the scope of the present paper. Therefore only forbidden lines will be used for comparison with observations of T Tauri stars, where the $\mathrm{H} \alpha$ line is contaminated by a strong magnetospheric contribution which will probably further affect the radiative transfer.

In order to adequately sample the innermost jet regions where strong gradients in density and velocity are present, we used a logarithmic sampling in $x=z / \varpi$ and in $\varpi_{0}$ between $\varpi_{\mathrm{i}}=0.07 \mathrm{AU}$ and $\varpi_{\mathrm{e}}=1 \mathrm{AU}$ and projected each cell onto a rectangular grid in the plane of the sky with $0.25 \mathrm{AU}$ pixel size. Maps were convolved with a $28 \mathrm{AU}(F W H M)$ Gaussian beam corresponding to $0.2^{\prime \prime}$ at the distance of Taurus, typical of resolutions accessible with adaptive optics or the Hubble Space Telescope.

To construct long slit spectra, line emission from each cell volume was distributed in radial velocity bins of $3.5 \mathrm{kms}^{-1}$, and summed perpendicular to the jet axis, keeping only the projected distance from the star, $d$, as spatial coordinate. The result was convolved with a $70 \mathrm{AU}$ $(F W H M)$ Gaussian along $d$ and with a $10 \mathrm{~km} \mathrm{~s}^{-1}(F W H M)$ Gaussian along the velocity axis, corresponding to typical resolutions achieved in ground-based long-slit spectra of microjets (e.g. Solf \& Böhm 1993; Lavalley-Fouquet et al. 2000, hereafter LF2000). 


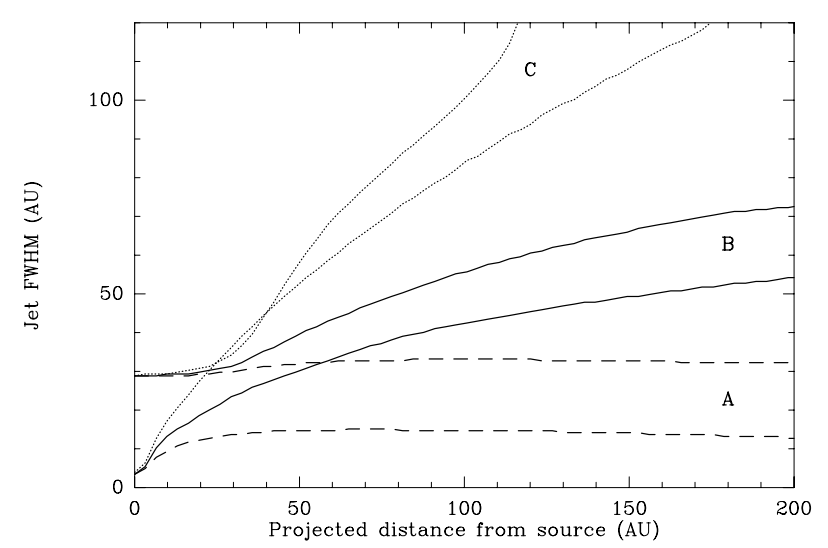

Fig. 1. Predicted jet FWHM (Gaussian fit) as a function of distance to the source for our models A, B, C (With $\xi=0.01,0.007$ and 0.005$)$ and two different beam sizes: $28 \mathrm{AU}$ and $2.8 \mathrm{AU}$, corresponding in Taurus to $0.2^{\prime \prime}$ and $0.02^{\prime \prime}$ (VLT diffraction limit). Note the strong dependence on $\xi$ and the considerable bias introduced by the beam size.

Note that qualitative forbidden line predictions for model A were presented by Cabrit et al. (1999) using a highly simplified temperature and ionization prescription for the plateau. Comparison of the present accurate results with this preliminary work will be used to probe the sensitivity of maps and line profiles on the underlying thermal and ionization structure.

\subsection{Wind morphology: Images and jet widths}

Synthetic emission maps are presented in Fig. 2 for our 3 models and typical parameters. They successfully reproduce two key features observed in microjets: (1) a strong, unresolved peak slightly shifted from the stellar position, (2) a jet-like body appearing collimated within 200 AU of the star. As in the X-wind model of Shu et al. (1994), this jet-like appearance is an "optical illusion" reflecting the collimation of the innermost, brightest streamlines; outer streamlines extend over a wider angle but have much lower surface brightness (cf. Fig. 1 in Cabrit et al. 1999). Note that the MHD disk wind solutions considered by Safier (1993a) did not collimate over such scales.

The shift of the unresolved peak from the star depends little on the value of $\xi$ or the chosen line, but decreases slightly for lower $\dot{M}_{\text {acc }}$, as heating then occurs sooner along each streamline (see Sect. 4 , Paper I). It is $\sim 5 \mathrm{AU}$ at $28 \mathrm{AU}$ resolution for $\dot{M}_{\text {acc }}=10^{-6} M_{\odot} \mathrm{yr}^{-1}$, smaller than observed (15-30 AU Solf \& Böhm 1993; Hirth et al. 1994a, 1997; Lavalley et al. 1997). However, this does not represent a reliable test of the model as observed shifts may be severely biased by inaccuracy in continuum subtraction (a $2 \%$ error in subtraction causes a $\simeq 4 \mathrm{AU}$ error for bright jets; Garcia et al. 1999) and by possible obscuration by a flared disk.

In contrast, Figs. 1 and 2 show that the jet width provides a powerful test of the models: it depends very little on the chosen line tracer, inclination, jet outer radius, and mass-accretion rate (at least within our explored range). It is also not overly sensitive to the detailed ionization and temperature structure, since we recover very similar widths as in the simplified calculations of Cabrit et al. (1999), for the same wind solutions. For this class of models, the jet FWHM depends mostly on the degree of opening of the innermost streamline, fixed by $\xi$ (Figs. 2, 1). Note that it is also severely biased by the beam size (Fig. 1), an example of the importance of proper convolution for comparison with observations.

Measurements of microjet widths at $0.1^{\prime \prime}-0.2^{\prime \prime}$ resolution were recently presented by (Burrows et al. 1996; Ray et al. 1996; Dougados et al. 2000a). Jets with no clear contamination by bowshock wings give strikingly consistent results, namely a typical jet $F W H M$ of $30-50 \mathrm{AU}$ at a distance of $200 \mathrm{AU}$. Models $\mathrm{A}$ and $\mathrm{B}$, with $\xi \simeq$ $0.007-0.01$, reproduce well these observations, while models with smaller $\xi<0.007$, in particular our model C, seem ruled out.

\subsection{Wind kinematics: Long-slit spectra and line profiles}

Long-slit spectra for our 3 models are presented in Fig. 2 for an inclination $60^{\circ}$ from pole-on and $\dot{M}_{\text {acc }}=$ $10^{-6} M_{\odot} \mathrm{yr}^{-1}$. Integrated line profiles for a wider range of inclinations and $\dot{M}_{\text {acc }}$ are presented in Fig. 3. The predicted profiles successfully reproduce several observed trends in TTS:

- Two velocity components are present: a low-velocity component (LVC) formed in very dense regions at the wind base, just where heating starts, and a high-velocity component (HVC), formed further up in the accelerated wind regions;

- The LVC is stronger in [O I] than in [N II], as observed (HEG95, Hirth et al. 1997): It is dominated by slowly rotating streamlines with low ionization fraction at $\varpi_{0} \simeq 1 \mathrm{AU}$

- The relative intensity of LVC versus HVC increases for lower $\dot{M}_{\text {acc }}$ (Fig. 3), as observed (Hartigan et al. 1995): This occurs because the hot plateau is reached sooner for lower accretion rates, increasing emission from the wind base and hence the LVC;

- The HVC is displaced further from the star than the $\mathrm{LVC}$ and the displacement is larger in $[\mathrm{NII}]$ than in [O I] (Fig. 2), as observed (Hirth et al. 1997). The [N II] profile peaks at the blue edge of the [O I] line profile (Fig. 3), as also observed (Hartigan et al. 1995; Hirth et al. 1997). This difference stems from the slow increase in ionization fraction with distance (cf. Fig. 2 of Paper I), which favors $\mathrm{N}$ II emission from the distant accelerated wind regions;

Long-slit spectra show apparent acceleration of the HVC, with the line profile peaking around $-100 \mathrm{~km} \mathrm{~s}^{-1}$ close to the star and around $-300 \mathrm{~km} \mathrm{~s}^{-1}$ at 150 AU. Similar gradients appear to be present in DG Tau and UY Aur (Solf \& Böhm 1993; Lavalley et al. 1997; Hirth et al. 1997). 

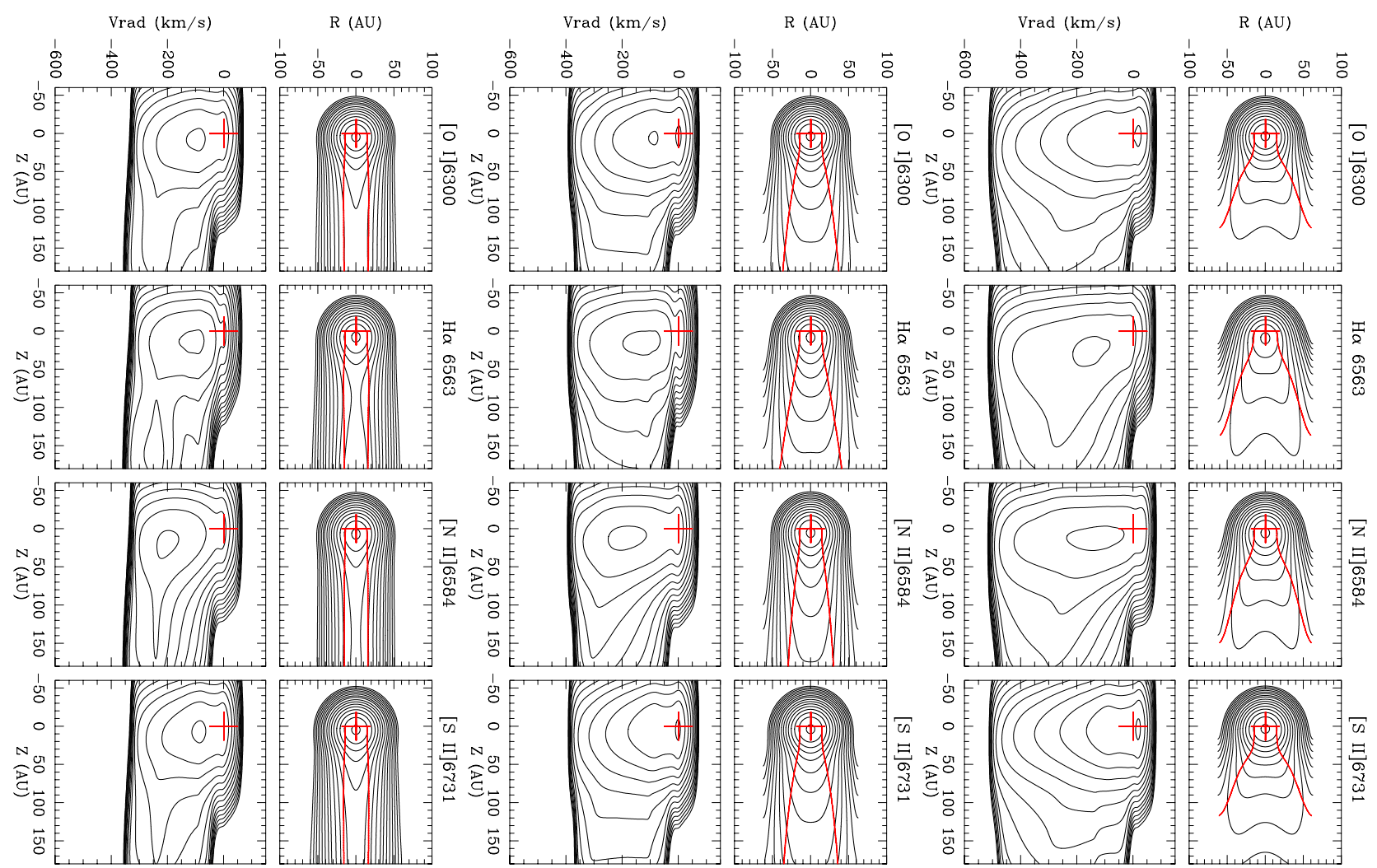

Fig. 2. Predicted maps and long-slit spectra in various emission lines for Models A, B, C ( $\xi=0.01,0.007$ and 0.005 left to right). Intensity maps are convolved with a $28 \mathrm{AU}$ beam, and thick grey lines plot the jet transverse $F W H M$ (Gaussian fit) as a function of position. Long-slit spectra along the jet axis are convolved by a $70 \mathrm{AU} \times 10 \mathrm{~km} \mathrm{~s}^{-1}$ point spread function, representative of current ground-based spectro-imaging performances. Inclination is $i=60^{\circ}$ from pole-on, and $\dot{M}_{\mathrm{acc}}=10^{-6} M_{\odot} \mathrm{yr}^{-1}$. A cross marks the stellar continuum position. Contours decrease by factors of 2 .

The predicted profiles fail, however, in two respects:

- The relative intensity of $\mathrm{LVC}$ versus $\mathrm{HVC}$ in $[\mathrm{OI}]$ is smaller than observed. The problem appears less severe in models with low $\xi \leq 0.005$ (see Model C in Fig. 3), as wider streamline expansion decreases the density in the outer wind and the resulting HVC flux. However, such models predict jet widths that are too wide (see Fig. 1 and Sect. 2.2 above). The weakness of the LVC in our models reflect the low temperatures encountered over most of the wind base, where low-velocity gas is located. Note that in the simplified calculations of Cabrit et al. (1999), which assumed $T=10^{4} \mathrm{~K}$ above $z / \varpi \simeq 0.7$, the LVC was more prominent, illustrating the crucial influence of temperature at the wind base on the resulting LVC intensity;

- Predicted maximum blue radial velocities in line profiles agree well with observations of the DG Tau microjet (e.g. Lavalley-Fouquet et al. 2000; Cabrit et al. 1999). However they appear too high compared with typical T Tauri stars, where $\left|V_{\text {blue }}\right| \leq 200 \mathrm{~km} \mathrm{~s}^{-1}$ (Hartigan et al. 1995; Hirth et al. 1997), unless most stars are observed at inclinations $\geq 80^{\circ}$ from pole-on (Fig. 3). Terminal velocities in the present class of cold disk wind models are $\simeq V_{\text {kep }}\left(\varpi_{0}\right) / \sqrt{\xi}$, so even our highest $\xi=0.01$ (model A) tends to give excessive speeds.

\subsection{Integrated line fluxes}

Figure 4 plots the predicted integrated luminosities in the $[\mathrm{O}$ I $] \lambda 6300$ and $[\mathrm{S}$ II $] \lambda 6731$ lines for our 3 models and our whole range of $\dot{M}_{\text {acc }}$ from $10^{-8}$ to $10^{-5} M_{\odot} \mathrm{yr}^{-1}$. For comparison, observed line luminosities in $\mathrm{T}$ Tauri stars with the same range in accretion rates, taken from Hartigan et al. (1995), are also plotted.

Two characteristics of the observations are well reproduced: (1) We find a correlation between [O I] and [S II] luminosities, with a slope close to that observed in TTS (Fig. 4a). (2) The [O I] line luminosity is seen to increase linearly with the accretion rate (Fig. 4b), a proportionality also observed in TTS (cf. Hartigan et al. 1995).

The latter proportionality is an interesting consequence of the opposite dependence of $n_{\mathrm{e}}$ and $T$ on $\dot{M}_{\text {acc }}$ in the hot plateau: as $\dot{M}_{\text {acc }}$ increases from $10^{-8}$ to $10^{-5} M_{\odot} \mathrm{yr}^{-1}, n_{\mathrm{e}}$ increases effectively only by a factor of 10 (recall that ionization fraction decreases almost as $1 / \dot{M}_{\text {acc }}$ ), while the plateau temperature decreases by a factor of a few (see Fig. 2 of Paper I). Given the strong temperature dependence of [O I] collisional excitation rates $q_{\text {lu }}$, the two effects cancel out and the emissivity $\left(\propto q_{\mathrm{lu}} n_{\mathrm{e}} n_{\mathrm{O}}\right.$ in the low-density regime) becomes proportional to the oxygen density only, i.e. to $\dot{M}_{\text {acc }}$. 

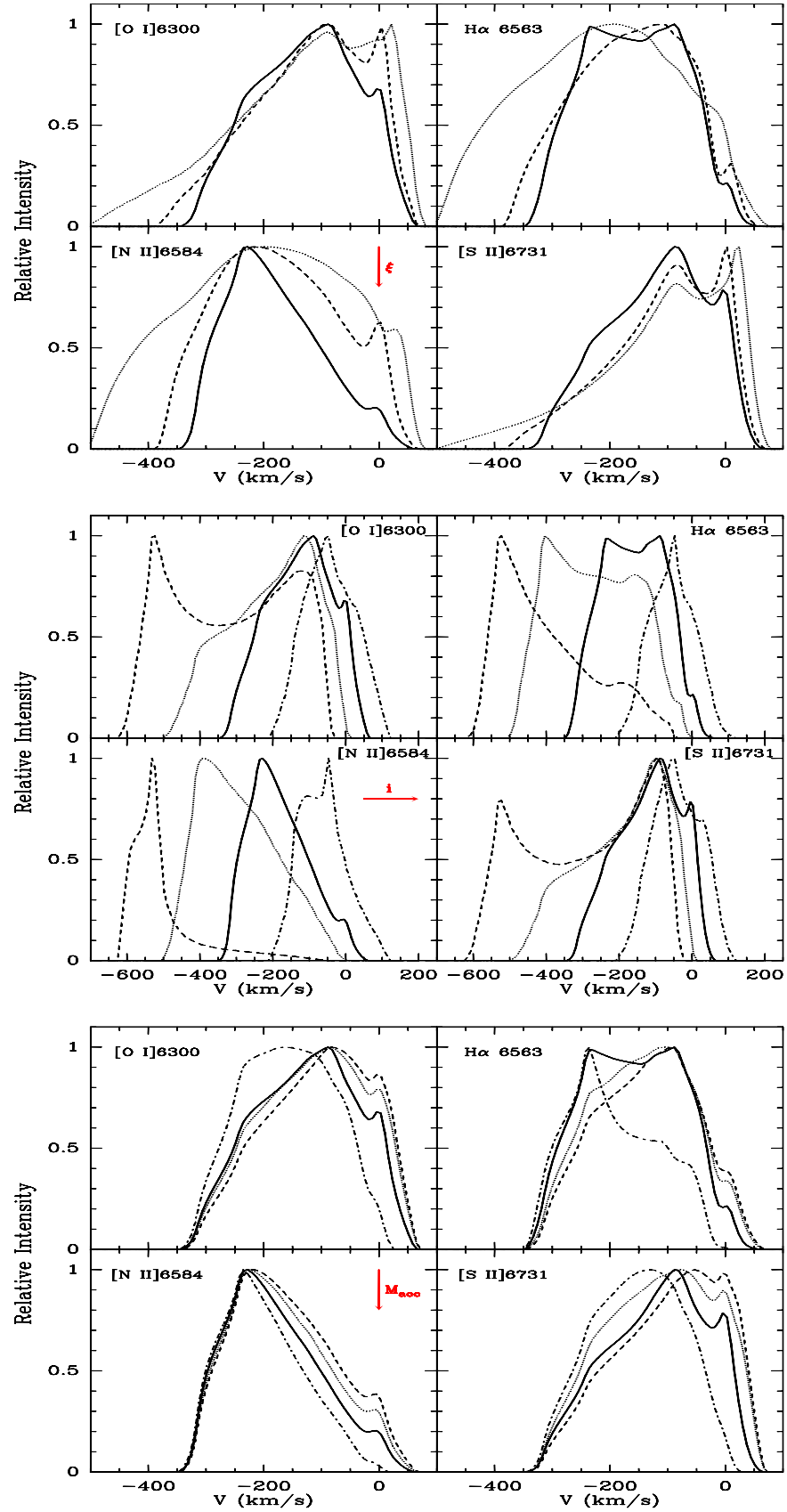

Fig. 3. Integrated line profiles behavior. Top: effect of ejection index $\xi$ : Model A ( $\xi=0.01$, solid $), \mathrm{B}(\xi=0.007$, dashed $)$ and $\mathrm{C}\left(\xi=0.005\right.$, dotted). Inclination is $i=60^{\circ}$ and accretion rate $\dot{M}_{\text {acc }}=10^{-6} M_{\odot} \mathrm{yr}^{-1}$. Middle: effect of inclination angle for Model A and $\dot{M}_{\text {acc }}=10^{-6} M_{\odot} \mathrm{yr}^{-1}: i=20^{\circ}$ (dashed), $i=40^{\circ}$ (dotted), $i=60^{\circ}$ (solid) and $i=80^{\circ}$ (dash-dot). Bottom: effect of accretion rate for Model $\mathrm{A}$ and inclination $i=60^{\circ}: \dot{M}_{\text {acc }}=10^{-8} M_{\odot} \mathrm{yr}^{-1}$ (dashed), $10^{-7} M_{\odot} \mathrm{yr}^{-1}$ (dotted), $10^{-6} M_{\odot} \mathrm{yr}^{-1}$ (solid) and $10^{-5} M_{\odot} \mathrm{yr}^{-1}$ (dash-dotted).

Quantitatively, however, the predicted fluxes fail in that they are systematically too weak compared to observations of TTS, by a factor 100 on average. We find $L([\mathrm{O} \mathrm{I}]) / L_{\odot} \simeq \dot{M}_{\mathrm{acc}} / M_{\odot} \mathrm{yr}^{-1}$. In this respect, we come to
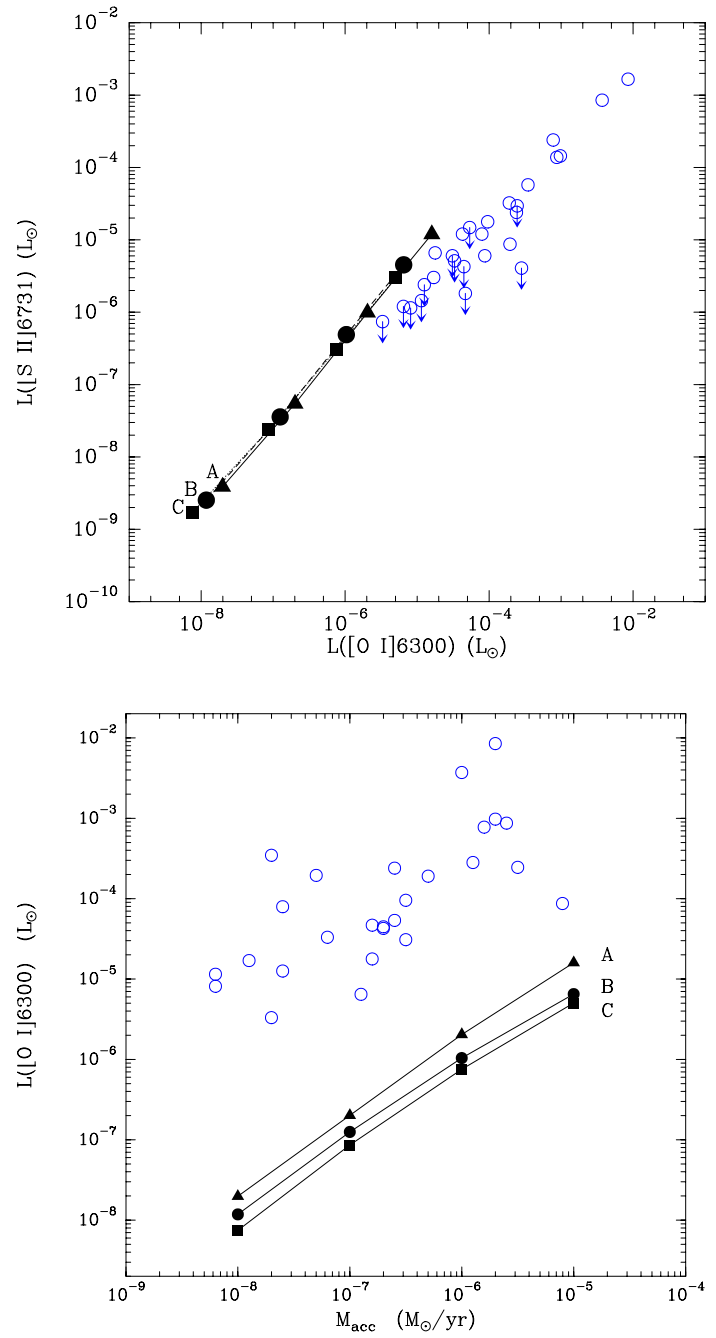

Fig. 4. Output fluxes for model A (triangles), model B (circles) and model $\mathrm{C}$ (squares). Model fluxes were integrated out to 200 AU from the star. Top: integrated luminosity in the $[\mathrm{S} \mathrm{II}] 6731$ line plotted against integrated luminosity in the $\left[\mathrm{O}_{\mathrm{I}}\right] 6300$ line. Accretion rates $\dot{M}_{\text {acc }}$ range from $10^{-8}$ to $10^{-5} M_{\odot} \mathrm{yr}^{-1}$ by factors of 10 (in the direction of increasing luminosity). Observed $\mathrm{T}$ Tauri star forbidden line luminosities from Hartigan et al. (1995) are shown as open circles, the arrows indicate upper limits on [S II]. Bottom: integrated luminosities in the [O I]6300 line plotted versus $\dot{M}_{\text {acc }}$ for our models. T Tauri stars total [O I] luminosities are shown as circles (Hartigan et al. 1995); only stars with detected emission are presented. Models predict a roughly linear law $L[\mathrm{O} \mathrm{I}] / L_{\odot} \simeq \dot{M}_{\mathrm{acc}} / M_{\odot} \mathrm{yr}^{-1}$, and lie typically a factor $\simeq 100$ below observations.

a very different conclusion from Safier (1993b), who concluded that his model was able to reproduce the range of forbidden line luminosities observed in TTS. There are two main reasons for this discrepancy: first, our lower rate coefficients for $\mathrm{H}-\mathrm{H}^{+}$momentum exchange result in a factor of 10 lower ionization fractions, $n_{\mathrm{e}}$ values, and hence [O I] luminosities, compared to Safier (Paper I). Second, Safier considered mass-loss rates ranging up to $10^{-6} M_{\odot} \mathrm{yr}^{-1}$, corresponding to a mass-accretion 
rate $\simeq 10^{-4} M_{\odot} \mathrm{yr}^{-1}$, i.e. an order of magnitude above the maximum $\dot{M}_{\text {acc }}$ values in TTS later deduced by HEG95 from veiling measurements.

In the next section, we show that the flux deficit in our models stems at least in part from a lower $n_{\mathrm{e}}$ than observed in TTS, but possibly also from an insufficient total density in our models.

\subsection{Forbidden line ratios and total densities}

The ratios of the $[\mathrm{OI}],[\mathrm{SII}]$, and [N II] forbidden lines provide powerful diagnostics of excitation conditions in a stellar jet (see e.g. Bacciotti \& Eislöffel 1999; Hartigan et al. 1994): [S II]6716/6731 is a decreasing function of the electronic density (until it reaches a "high-density limit" $\simeq 0.45$ when $n_{\mathrm{e}} \gg 10^{4} \mathrm{~cm}^{-3}$, the critical density of the $[\mathrm{SII}] 6716$ line); $[\mathrm{NII}] /[\mathrm{OI}]$ is an increasing function of the electronic fraction $f_{\mathrm{e}}$; and $[\mathrm{S} \mathrm{II}] /[\mathrm{OI}]$ decreases with increasing temperature ${ }^{1}$. Hence, these line ratios provide a test of the heating/ionization mechanism in the flow, though they do not test the underlying dynamical solution.

In particular, LF2000 and Lavalley (2000) showed how diagnostic diagrams based on these line ratios (see their Fig. 3) could be used to separate various proposed heating mechanisms, such as shocks, mixing-layers, and ambipolar diffusion heated MHD disk winds. For the latter case, LF2000 used preliminary results from the present work. We plot in Fig. 5 our complete set of results using the same line ratio diagrams.

Each curve in Fig. 5, corresponding to a given wind model and mass accretion rate, shows the evolution of line ratios with distance from the star from $0 \mathrm{AU}$ to $200 \mathrm{AU}$, after convolution of jet images by a 70 AU beam and summation perpendicular to the jet axis (note that LF2000 plotted ratios out to a distance of $600 \mathrm{AU}$, for comparison with observations of DG Tau, and used calculations with no depletion on dust, which affects the $[\mathrm{S}$ II $] /[\mathrm{O} \mathrm{I}]$ ratio).

Spatially resolved line ratios within 200 AU of the star are available for only four TTS microjets so far: HH 30, Th 28, DG Tau, and RW Aur (Bacciotti \& Eislöffel 1999; Lavalley-Fouquet et al. 2000; Bacciotti et al. 2000; Bacciotti 2000; Dougados et al. 2000b). Comparison with our predictions, at the same beam resolution and distance from the star, indicates that our models have slightly too high temperatures but insufficient beam-averaged electronic density and fractional ionization. In particular, the observed [S II] 6716/6731 towards the star is always in the high-density limit $\simeq 0.45$, indicating $n_{\mathrm{e}} \geq 2 \times 10^{4} \mathrm{~cm}^{-3}$, while our models predict higher ratios at the stellar position corresponding to a beam-averaged $n_{\mathrm{e}} \simeq 0.6-2 \times$ $10^{3} \mathrm{~cm}^{-3}$. This difference of at least a factor $10-30$ readily explains a substantial fraction of the flux deficit noted

\footnotetext{
1 The latter two ratios also decrease with increasing $n_{\mathrm{e}}$ when $n_{\mathrm{e}}$ exceeds the critical density of the line in the numerator.
}

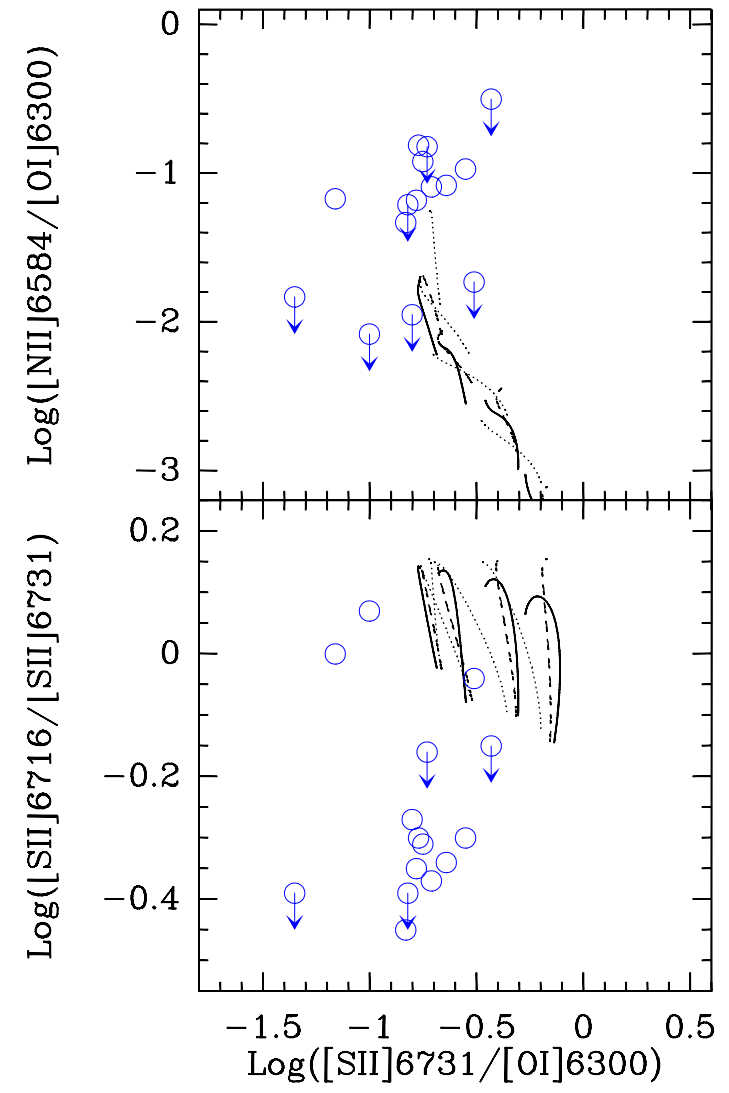

Fig. 5. Line ratio diagrams for Model A (solid), B (dashed) and C (dotted). Each curve refers to a given $\dot{M}_{\text {acc }}$, and connects line ratios at various distances along the jet, from $z=-50 \mathrm{AU}$ to $200 \mathrm{AU}$ (after convolution by a $70 \mathrm{AU}$ beam and summation perpendicular to the jet axis). Distance increases with [S II]6716/6731 and with [N II]/[O I] (bottom to top in both figures). Accretion rates vary from $\dot{M}_{\text {acc }}=10^{-8}$ to $10^{-5} M_{\odot} \mathrm{yr}^{-1}$ in factors of 10 from left to right in both figures.

above in Sect. 2.4. Two further important remarks are in order:

(1) We stress that, because of volume effects and strong density gradients, beam-averaged line ratios differ greatly from local line ratios. In particular they give very little weight to the inner densest parts of the wind, where the [S II] 6716/6731 ratio is locally in the high-density limit. Hence it is essential to apply proper convolution by the observing beam to perform meaningful comparison with observed ratios.

(2) All predicted line ratios, in particular the highly ionization sensitive $[\mathrm{NII}] /[\mathrm{OI}]$, would agree much better with observations if one includes extra mechanical energy deposition in shocks, as demonstrated in LF2000 (see also Dougados et al. 2000b; Lavalley 2000), although this would involve additional free parameters (e.g. ratio of shock speed to wind speed, magnetic field limiting the postshock compression).

For the latter reason, as noted by Bacciotti \& Eislöffel (1999), the total density $n_{\mathrm{H}}=n_{\mathrm{e}} / f_{\mathrm{e}}$ is a more fundamental quantity that can be directly compared with MHD model predictions independently of the ionization 
process (although $n_{\mathrm{H}}$ can still be affected by shock compression and by beam averaging). Out of the 4 abovementioned T Tauri stars with spatially resolved line ratios, only two (DG Tau and RW Aur) have estimates of disk accretion rates, both of order $10^{-6} M_{\odot} \mathrm{yr}^{-1}$ (HEG95). Observed values of $n_{\mathrm{H}}$ as a function of distance (LF2000; Dougados et al. 2000b) are 10-100 times higher than total densities on the innermost streamline for our models with the same $\dot{M}_{\text {acc }}$ (see Fig. 1 of Paper I). While shock compression could account for a factor of 10 Hartigan et al. (1994), it seems that our models may still have too low density close to the star.

As a word of caution, we note that the above two stars are the strongest forbidden line emitters in the HEG95 sample and may therefore not be representative of wind physical conditions in an average TTS. More sensitive observations would be needed to probe $n_{\mathrm{e}}$ and total density $n_{\mathrm{H}}$ in objects of weaker wind emission and lower accretion rates.

\section{Summary and conclusion}

In a companion paper Paper I, we computed the thermal and ionization structures of self-similar, magneticallydriven disk winds heated by ambipolar diffusion. In this paper, we compared our model predictions in forbidden lines with all existing observational constraints in T Tauri microjets, namely: (1) jet images; (2) jet widths; (3) longslit spectra and integrated line profiles; (4) line fluxes; and (5) line ratios and total densities. Jet widths, line profiles, and total densities provide powerful tests of the jet dynamics and collimation, whereas line ratios and fluxes are strongly influenced by the underlying heating assumptions. Given the steep gradients in physical conditions in the jet, beam convolution is found to have a crucial effect on model predictions, and we stress that it is an essential step for a meaningful comparison with observations.

The model reproduces several observed trends: (1) images show an unresolved peak and an extended highvelocity jet, of width compatible with observations; (2) line profiles present a low-velocity component (LVC), compact and near the star, and a high-velocity component (HVC), tracing the jet. These components are not an inclination effect but intrinsic to the jet dynamics. (3) The systematic differences between [O I] and [N II] profiles are reproduced; (4) line fluxes are proportional to $\dot{M}_{\text {acc }}$; and (5) the $[\mathrm{O} \mathrm{I}]-[\mathrm{S}$ II $]$ correlation slope is recovered.

The model also encounters several problems: (1) the line ratios ([S II]6716, 6731, [N II]/[O I] ) are not well reproduced, and indicate insufficient ionization in the model; (2) line fluxes are too low by a factor of 100, and electronic densities by a factor of at least 10-30; (3) the relative intensity of the low-velocity component (LVC), present in our profiles and formed in the inner wind regions just above the disk, is insufficient; (4) wind terminal velocities appear too high, and total densities at the wind base appear too small, when compared with current estimates in 2 bright microjets.
Both line intensity and line ratio problems can be readily solved by introducing an extra ionizing mechanism, e.g. in shocks (Lavalley-Fouquet et al. 2000; Dougados et al. 2000b). However, terminal wind velocities and total densities would not be affected. As a consequence, cold selfsimilar MHD disk wind models with isothermal magnetic surfaces seem to be ruled out by observations of T Tauri microjets. In the context of magnetized accretion-ejection models considered here, a possible improvement would be to relax the assumption of isothermal magnetic surfaces. Indeed, very efficient ejection (namely $\xi$ up to 0.5 ) can be attained by allowing for some entropy injection at the disk surface Casse \& Ferreira (2000). Such models are very promising for they naturally provide both higher jet densities and lower terminal velocities (see Fig. 8 in Casse $\&$ Ferreira 2000). In this respect, we independently support Kwan 1997's point of view that active disk chromospheres may be necessary ingredients in T Tauri circumstellar disks.

Acknowledgements. P. J. V. G. acknowledges financial support from Fundação para a Ciência e Tecnologia by the PRAXIS XXI/BD/5780/95, PRAXIS XXI/BPD/20179/99 grants. The work of LB was supported by the CONACyT grant 32139-E. We thank the referee, Pedro N. Safier, for his helpful comments. We also acknowledge fruitful discussions with Francesca Bacciotti, Catherine Dougados, Pierre Ferruit, Eliana Pinho, Alex Raga, and Eric Thiébaut. P. J. V. G. warmly thanks the Airi team and his adviser, Renaud Foy, for their constant support.

\section{References}

Bacciotti, F. 2000, in Emission Lines from Jet Flows, Isla Mujeres, November 13-17, 2000, E2

Bacciotti, F., \& Eislöffel, J. 1999, A\&A, 342, 717

Bacciotti, F., Mundt, R., Ray, T. P., Eislöffel, J., Solf, J., \& Camezind, M. 2000, ApJL, 537, L49

Burrows, C. J., Stapelfeldt, K. R., Watson, A. M., et al. 1996, ApJ, 473, 437

Cabrit, S., Edwards, S., Strom, S. E., \& Strom, K. M. 1990, ApJ, 354, 687

Cabrit, S., Ferreira, J., \& Raga, A. C. 1999, A\&A, 343, L61

Casse, F., \& Ferreira, J. 2000, A\&A, 361, 1178

Cohen, M., Emerson, J. P., \& Beichman, C. A. 1989, ApJ, 339, 455

Dougados, C., Cabrit, S., Lavalley, C., \& Ménard, F. 2000a, A\&A, 357, L61

Dougados, C., Cabrit, S., \& Lavalley-Fouquet, C. 2000b, in Emission Lines from Jet Flows, Isla Mujeres, November 13-17, 2000, E2

Ferreira, J. 1997, A\&A, 319, 340

Garcia, P. J. V., Ferreira, J., Cabrit, S., \& Binette, L. 2001, A\&A, 377, 589, Paper I

Garcia, P. J. V., Thiébaut, E., \& Bacon, R. 1999, A\&A, 346, 892

Gullbring, E., Hartmann, L., Briceno, C., \& Calvet, N. 1998, ApJ, 492, 323

Hartigan, P., Edwards, S., \& Ghandour, L. 1995, ApJ, 452, 736 
Hartigan, P., Morse, J. A., \& Raymond, J. 1994, ApJ, 436, 125

Hirth, G. A., Mundt, R., \& Solf, J. 1994a, A\&A, 285, 929

Hirth, G. A., Mundt, R., Solf, J., \& Ray, T. P. 1994b, ApJL, 427, L99

Hirth, G. A., Mundt, R., \& Solf, J. 1997, A\&AS, 126, 437

Kepner, J., Hartigan, P., Yang, C., \& Strom, S. 1993, ApJ, 415, L119

Kwan, J. 1997, ApJ, 489, 284

Lavalley, C. 2000, Etude de la morphologie et de la cinématique de l'émission des raies interdites autour des étoiles T Tauri (Université Joseph Fourier - Grenoble 1), Thesis

Lavalley, C., Cabrit, S., Dougados, C., Ferruit, P., \& Bacon, R. 1997, A\&A, 327, 671
Lavalley-Fouquet, C., Cabrit, S., \& Dougados, C. 2000, A\&A, 356, L41

Ray, T. P., Mundt, R., Dyson, J. E., Falle, S. A. E. G., \& Raga, A. C. 1996, ApJL, 468, L103

Safier, P. N. 1993a, ApJ, 408, 115

Safier, P. N. 1993b, ApJ, 408, 148

Shang, H., Shu, F. H., \& Glassgold, A. E. 1998, ApJL, 493 L91

Shu, F., Najita, J., Ostriker, E., Wilkin, F., Ruden, S., \& Lizano, S. 1994, ApJ, 429, 781

Solf, J. 1989, in Low Mass Star Formation and Pre-main Sequence Objects, 399

Solf, J., \& Böhm, K. H. 1993, ApJL, 410, L31 